\title{
利用微粒过滤器同时催化去除柴油机微粒和 $\mathrm{NO}_{x}$
}

刘光辉 黄震 上官文峰“ 阎存仙

(上海交通大学机械与动力工程学院, 上海 200030.* 联系人, E-mail: shangguan@ @stu.edu.cn)

摘要 通过在柴油机微粒过滤器( DPF) 上涂覆复合金属氧化物催化剂 $\mathrm{Cu}_{0.95} \mathrm{~K}_{0.05} \mathrm{Fe}_{2} \mathrm{O}_{4}$, 对同时催化去除 柴油机排放的 $\mathrm{NO}_{x}$ 和微粒 $(\mathrm{PM})$ 反应特性及机理进行了研究. 在程序升温反应过程中观察到 $\mathrm{NO}_{x}$ 的还原 和 $\mathrm{CO}_{2}$ 的形成出现在相同的温度范围内, 证实了在柴油机排气氧化气氛中同时去除 $\mathrm{NO}_{x}$ 和 $\mathrm{PM}$ 反应的 发生. 柴油机微粒中的可溶有机成分(SOF) 和固体碳粒(soot) 分别在较低的温度和较高的温度下还原 $\mathrm{NO}_{x}, \mathrm{NO}_{x}$ 浓度降低了 $18 \%$ 左右. 在催化剂作用下, $\mathrm{PM}$ 的燃烧温度下降了 $150^{\circ} \mathrm{C}$ 以上. 微粒在不同气氛中 的催化氧化燃烧活性为 $\mathrm{NO}+\mathrm{O}_{2}>\mathrm{O}_{2}>\mathrm{NO}$. 该方法集 $\mathrm{PM}$ 的捕集、 $\mathrm{PM}$ 的氧化燃烧和 $\mathrm{NO}_{x}$ 的还原等功 能于一体, 是一种有发展前途的柴油机排放后处理新技术.

关键词 柴油机 氮氧化物 微粒 同时去除 催化

柴油机以其高的燃油经济性、高可靠性、低维护 成本的特点, 大规模地用作为汽车运输动力. 然而, 在近十几年来, 以柴油机排放引起的环境污染越来 越严重. 目前柴油机排放法规对柴油机的排放, 尤其 对 $\mathrm{NO}_{x}$ 和微粒 $(\mathrm{PM})$ 的排放, 提出了更严格的要求. 由 于通过单一的机内改良措施难以同时把 $\mathrm{NO}_{x}$ 和 $\mathrm{PM}$ 降低到符合严格的排放标准的要求, 因此必须开发 后处理技术, 以有效地降低柴油机的污染排放.

$\mathrm{NH}_{3}$ 能在 $\mathrm{O}_{2}$ 存在的情况下选择性地还原 $\mathrm{NO}_{x}$, 因此用 $\mathrm{NH}_{3}$ 来催化还原 $\mathrm{NO}_{x}$ 的选择催化还原技术 (selective catalytic reduction, SCR) 已经广泛投入使 用 ${ }^{[1,2]}$. 但是, 由于 $\mathrm{NH}_{3}$ 的毒性和储存的问题, 这项技 术主要应用在固定源柴油机, 而不适合于车用发动 机. 在对柴油机排放中的微粒(主要由黑烟和可溶性 有机物组成)的净化方面, 近些年来人们研究了诸如 泡沫陶瓷、蜂窝陶瓷载体和金属丝网等不同类型的微 粒捕集器. 然而, 若集聚在过滤器上的微粒未被及时 地除去, 将导致柴油机背压增大, 从而影响柴油机的 性能. 催化剂的使用可以有效地降低微粒的燃点, 使 微粒在较低的温度下燃烧, 从而能降低微粒过滤器 两端的压力差. 这项技术目前在世界上已被广泛地 研究 ${ }^{[3-5]}$. Johnson Matthey 开发的一种柴油机微粒的 连续再生捕集 $(\mathrm{CRT})$ 技术, 证明了 $\mathrm{NO}_{2}$ 和微粒之间的 反应. Yoshida 等人 ${ }^{[6]}$ 提出了 “soot- $\mathrm{O}_{2}$-NO” 三组分之间 反应的可能性. 这些工作促进了 Teraoka 和 Shangguan 等人对同时去除柴油机中 $\mathrm{NO}_{x}$ 和固体干碳粒 (dry soot) 的一系列基础性研究 ${ }^{[7 ~ 13]}$. 这些研究采用了程序温度 反应(TPR)技术, 考察了 “催化剂-干碳粒”紧密混合 物(tight soot/catalyst mixture) 在 $\mathrm{O}_{2}-\mathrm{NO}$ 气氛中的催化 反应特性. 研究结果证明了钙钛矿型 ${ }^{[7,13]}$ 和尖晶石 型 ${ }^{[8,10]}$ 等复合金属氧化物有望用于柴油机尾气中 PM 和 $\mathrm{NO}_{x}$ 的同时催化去除.

为进一步探讨在实际柴油机中 $\mathrm{PM}-\mathrm{NO}_{x}$ 同时催化 净化的可行性, 本文采用壁流式蜂窝陶瓷微粒过滤器 (DPF) 作为催化载体, 制得催化剂 $\left(\mathrm{Cu}_{0.95} \mathrm{~K}_{0.05} \mathrm{Fe}_{2} \mathrm{O}_{4}\right)$ DPF 复合体, 研究了实际柴油机排放的微粒 $(\mathrm{PM})$ 在 $\mathrm{NO}_{x}-\mathrm{O}_{2}$ 环境中的催化反应特性, 并且讨论了催化作 用机理.

\section{1 实验}

在原先合成的众多催化材料中, $\mathrm{Cu}_{0.95} \mathrm{~K}_{0.05} \mathrm{Fe}_{2} \mathrm{O}_{4}$ 在 “催化剂-干碳粒” 紧密混合物的 TPR 中表现为是 一种比较理想的 PM-NO 同时催化材料 ${ }^{[10]}$. 因此, 本 研究首先以原先同样的方法制得 $\mathrm{Cu}_{0.95} \mathrm{~K}_{0.05} \mathrm{Fe}_{2} \mathrm{O}_{4}$ 粉 末, 再将其负载于 DPF 上, 从而获得催化剂-DPF 复 合体. 其工艺过程和方法如下, DPF 采用壁流式蜂窝 陶瓷过滤器(景德镇特种陶瓷材料研究所定制), 它的 直径和长度分别为 45 和 $70 \mathrm{~mm}$, 具有 100 孔/平方英寸 $(1$ 英寸 $=2.54 \mathrm{~cm})$ 结构. DPF 先用 $\mathrm{Al}_{2} \mathrm{O}_{3}$ 溶胶浸渍 $2 \mathrm{~h}$, 在 $480^{\circ} \mathrm{C}$ 下加热 $2 \mathrm{~h}$ 形成 $\gamma-\mathrm{Al}_{2} \mathrm{O}_{3}$ 中间层(质量分数约 $2.1 \%)$. 涂覆有 $\gamma-\mathrm{Al}_{2} \mathrm{O}_{3}$ 中间层的 DPF浸渍在浓度质量分 数为 $8.0 \%$ 的 $\mathrm{Cu}_{0.95} \mathrm{~K}_{0.05} \mathrm{Fe}_{2} \mathrm{O}_{4}$ 催化剂悬浊液中 $2 \mathrm{~h}$, 然后 在 $600^{\circ} \mathrm{C}$ 下灼烧 $2 \mathrm{~h}, \mathrm{DPF}$ 上催化剂的负载量约为 $1.6 \mathrm{~g}$. 将这种催化剂/DPF 放人 2135 型柴油机的排气管中, 通 过控制油门的大小采集微粒, 得到约为 $750 \mathrm{mg}$ 的挂烟 1 量(它同时含有 $\mathrm{SOF}$, ${ }^{1}$ 水等). 挂烟后的 DPF 放人固定床 
流动反应器 (内径为 $50 \mathrm{~mm}$ 的石英管)进行程序温度 反应. 在反应前, 用真空洜将反应系统抽真空, 以消 除空气对系统的影响, 然后通高纯氦气体 $1 \mathrm{~h}$, 在用 气相色谱测不出 $\mathrm{N}_{2}$ 和 $\mathrm{O}_{2}$ 之后, 再在 $100^{\circ} \mathrm{C}$ 下保持 $1 \mathrm{~h}$. 反应气体为 $\mathrm{O}_{2}(5.0 \%)-\mathrm{NO}\left(2600 \times 10^{-6}\right)-\mathrm{He}$ (平衡气), 它由 $\mathrm{O}_{2}(10 \%) / \mathrm{He}, \mathrm{NO}(0.5 \%) / \mathrm{He}$ 和纯 $\mathrm{He}$ 混合稀释而成. 反应系统以 $500 \mathrm{~mL} / \mathrm{min}$ 的气体总流量和 $1.5^{\circ} \mathrm{C} / \mathrm{min}$ 的升温速率进行程序升温加热. 系统出口气体每隔 25 min 用热导气相色谱仪(海信 GC-920)进行分析, $\mathrm{N}_{2}$ 和 $\mathrm{O}_{2}$ 采用分子篮 $5 \mathrm{~A}$ 分离柱进行分离, $\mathrm{CO}_{2}$ 通过 Porapak QS 分离柱进行分离; $\mathrm{NO}_{x}$ 采用盐酸荎乙二胺 分光光度法进行分析.

\section{2 结果和讨论}

催化剂-DPF 复合体中的中间层起到提高比表面 积和增强催化剂颗粒-DPF 结合性的作用. BET 比表 面积分析结果表明, 由于 $\gamma-\mathrm{Al}_{2} \mathrm{O}_{3}$ 中间层的涂覆, 使 得 DPF 的比表面积由 $0.78 \mathrm{~m}^{2} / \mathrm{g}$ 增加到 $3.88 \mathrm{~m}^{2} / \mathrm{g}$. 图 1 和 2 分别为涂覆了催化剂颗粒的载体表面和内部的 SEM 图像(S-520 SEM HITACHI). 载体内部的 SEM 图像是在 DPF 壁截面上观察到的. 可以看到催化剂 颗粒均匀地被覆于载体表面, 并且少数催化剂颗粒 通过孔隙进人了 DPF 的结构内部(图 2). XRD(Rigaku Dmax-rC) 测定结果确认了所制得各种氧化物的晶体 结构.

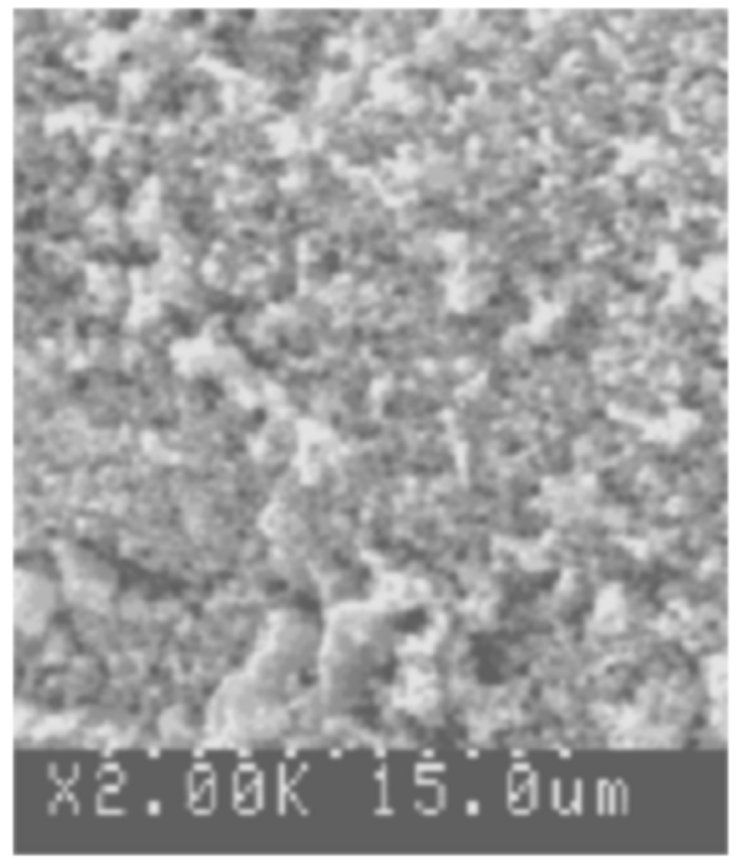

图 1 涂覆在 DPF 表面的催化剂SEM照片

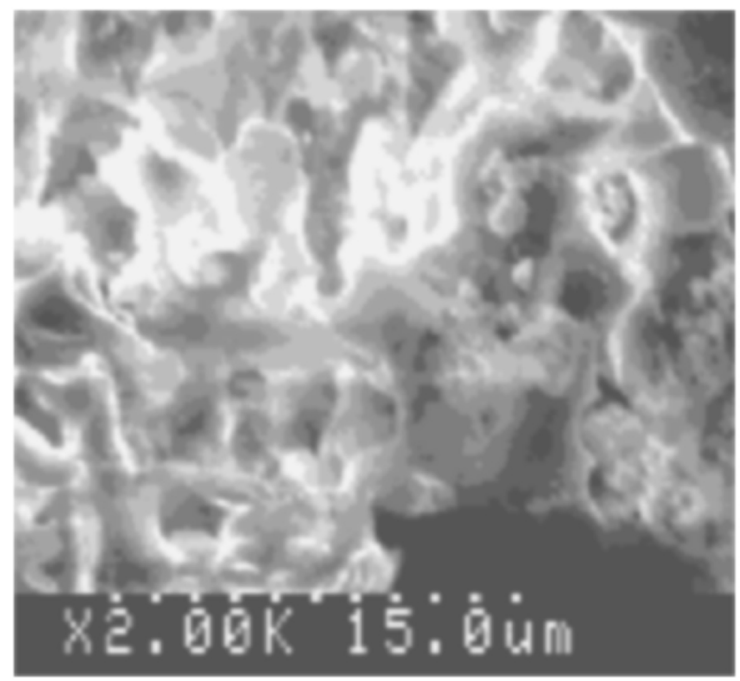

图 2 涂覆催化剂的 DPF 的内部组织 SEM 照片

图 3 是涂覆有 $\mathrm{Cu}_{0.95} \mathrm{~K}_{0.05} \mathrm{Fe}_{2} \mathrm{O}_{4}$ 催化剂的 DPF 的 程序温度反应结果. 从图中可以看出, $\mathrm{CO}_{2}$ 的出现和 $\mathrm{NO}_{x}$ 的降低在 150 和 $600{ }^{\circ} \mathrm{C}$ 之间的相同范围产生. 不 难发现, 该图中的曲线形状和原先 “催化剂-干碳粒” 紧密混合物的反应曲线 ${ }^{[7 \sim 9]}$ 明显不同. 原先的干碳粒 成分单一, 因此仅出现一个峰. 本文程序升温曲线的 复杂性是由于实际柴油机微粒组成的复杂性所致的. 出现在 $385^{\circ} \mathrm{C}$ 的第 1 个 $\mathrm{CO}_{2}$ 峰是由于微粒中可溶性有 机物成分 (SOF) 的燃烧形成的, 出现在 $516^{\circ} \mathrm{C}$ 的第 2 个 $\mathrm{CO}_{2}$ 峰是由于微粒中干碳粒成分的燃烧形成的. 由 $150^{\circ} \mathrm{C}$ 开始 $\mathrm{NO}_{x}$ 浓度降低的结果可以看出, 可溶性有 机物成分的燃烧也明显地降低了 $\mathrm{NO}_{x}$. 也就是说, 在 低温下 $\mathrm{NO}_{x}$ 被可溶性有机物 $\mathrm{SOF}\left(\mathrm{C}_{x} \mathrm{H}_{y} \mathrm{O}_{z}\right)$ 还原, 在高 温下被碳粒 $(\mathrm{C})$ 还原, 化学反应如下:

$$
\begin{gathered}
\mathrm{C}_{x} \mathrm{H}_{y} \mathrm{O}_{z}+\mathrm{NO}+\mathrm{O}_{2} \rightarrow \mathrm{CO}_{2}+\mathrm{N}_{2}+\mathrm{H}_{2} \mathrm{O} \\
\mathrm{C}+\mathrm{NO}+\mathrm{O}_{2} \rightarrow \mathrm{CO}_{2}+\mathrm{N}_{2}
\end{gathered}
$$

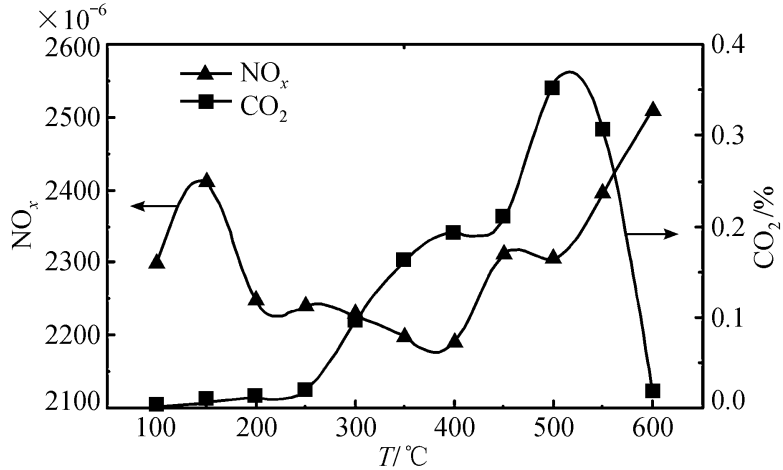

图 3 涂覆有 $\mathrm{Cu}_{0.95} \mathrm{~K}_{0.05} \mathrm{Fe}_{2} \mathrm{O}_{4}$ 催化剂的 DPF 的程序升温 反应结果 
最终在高温下 $\mathrm{CO}_{2}$ 浓度急剧降低是由于 DPF 上所挂 的微粒已经燃烧完毕, 从而使 $\mathrm{NO}_{x}$ 在 $600^{\circ} \mathrm{C}$ 以上恢复 到它的初始浓度 $\left(2600 \times 10^{-6}\right)$. 另外需要指出的是, 反应初始时 $\mathrm{NO}_{x}$ 浓度小于 $2600 \times 10^{-6}$ 是由于 $\mathrm{NO}_{x}$ 反 应系统的吸附尚未达到完全饱和所引起的.

为了进一步考证同时去除柴油机 $\mathrm{NO}_{x}$ 和 $\mathrm{PM}$ 的 催化剂的有效性, 在 DPF 不涂覆催化剂的情况下挂烟, 并进行同样的程序升温反应实验. 图 4 和 5 是在相同 的气体流量和成分 $\left(\mathrm{NO}\right.$ 为 $2600 \times 10^{-6}$ )下, $\mathrm{NO}_{x}$ 和 $\mathrm{CO}_{2}$ 浓度变化曲线的比较. 可以发现, 在 $200 \sim 400^{\circ} \mathrm{C}$ 的温度 范围内, DPF 涂覆催化剂的情况下, $\mathrm{NO}_{x}$ 浓度比不涂 覆催化剂的情况大约低 $450 \times 10^{-6}$, 下降了 $18 \%$ 左右. 在 DPF 不涂覆催化剂的情况下, $100 \sim 200^{\circ} \mathrm{C}$ 温度范 围内, $\mathrm{NO}_{x}$ 浓度的增大是由于低温下被系统吸附的 $\mathrm{NO}_{x}$ 脱附所产生的.

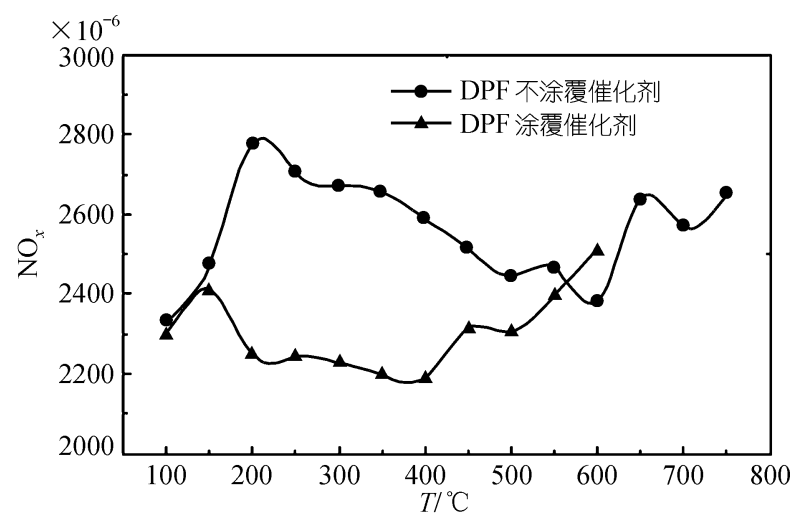

图 4 DPF 分别在涂覆和不涂覆催化剂情况下进行程序 升温反应 $\mathrm{NO}_{x}$ 浓度的比较

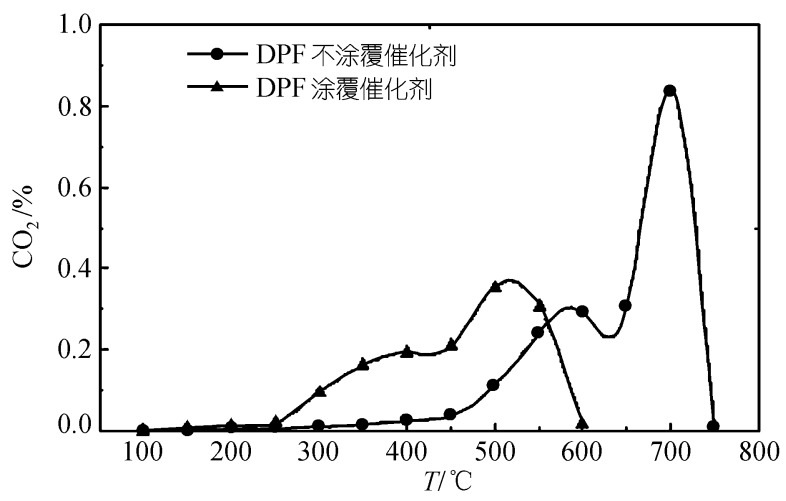

图 $5 \mathrm{DPF}$ 分别在涂覆和不涂覆催化剂情况下进行程序 升温反应 $\mathrm{CO}_{2}$ 浓度的比较 由图 5 程序升温反应的 $\mathrm{CO}_{2}$ 浓度变化比较可以 $\mathrm{dol} / 1$ 度反应所得的 $T_{\mathrm{ig}}\left(\text { 约 } 265^{\circ} \mathrm{C}\right)^{[10]}$ 要高, 这主要是由于催
看出，在 DPF涂覆催化剂时，微粒的燃烧温度明显比 不涂覆催化剂时低. 根据以前的研究 ${ }^{[7,8,13]}$, 用干碳 粒的点火温度 $\left(T_{\mathrm{ig}}\right)$ 和 $\mathrm{NO}_{x}$ 的转换率来评估催化反应 的性能，而 $T_{\mathrm{ig}}$ 是根据干碳粒的燃烧所形成的 $\mathrm{CO}_{2}$ 曲 线上切线与零浓度相交的温度点来确定的. 对 DPF 涂覆催化剂和不涂覆催化剂的反应情况下, 分别根 据以 516 和 $700{ }^{\circ} \mathrm{C}$ 为峰值的 $\mathrm{CO}_{2}$ 曲线所判断得到的碳 粒的起燃温度 $\left(T_{\mathrm{ig}}\right)$ 分别为 380 和 $610^{\circ} \mathrm{C}$. 同时发现 SOF 的起燃温度也明显降低. 这说明催化剂能有效 地降低微粒的燃烧温度.

为了考察 $\mathrm{NO}$ 和 $\mathrm{O}_{2}$ 在同时催化去除柴油机 $\mathrm{NO}_{x}$ 和 $\mathrm{PM}$ 反应中的作用, 本研究进行了 $\mathrm{NO}\left(2600 \times 10^{-6}\right)$ 和 $\mathrm{O}_{2}(5 \%)$ 共存、只有 $\mathrm{NO}\left(2600 \times 10^{-6}\right)$ 存在以及只有 $\mathrm{O}_{2}(5 \%)$ 存在时的程序升温反应的比较. 从图 6 的 $\mathrm{CO}_{2}$ 浓度曲线可以看出, SOF 和碳粒的起燃温度、燃尽温 度在 $\mathrm{NO}, \mathrm{O}_{2}$ 和 $\mathrm{NO}+\mathrm{O}_{2}$ 的气氛下依次降低. SOF 的起 燃温度在 3 个反应中依次为 310,280 和 $240^{\circ} \mathrm{C}$, 碳粒 的燃尽温度在 3 个反应中依次为 850,740 和 $600^{\circ} \mathrm{C}$. 可见, $\mathrm{NO}$ 和 $\mathrm{O}_{2}$ 的共存能提高 SOF 和碳粒的催化燃烧 活性. 这是因为在 $\mathrm{NO}$ 和 $\mathrm{O}_{2}$ 共存气氛中, 在催化剂的 作用下, $\mathrm{NO}$ 转化为 $\mathrm{NO}_{2}$, 而 $\mathrm{NO}_{2}$ 比 $\mathrm{O}_{2}$ 的氧化能力更 强, 形成具有高活性的 $\mathrm{C}^{*}[\mathrm{O}]$ 中间体, 从而促进了 SOF 和碳粒的氧化燃烧 ${ }^{[9,12]}$.

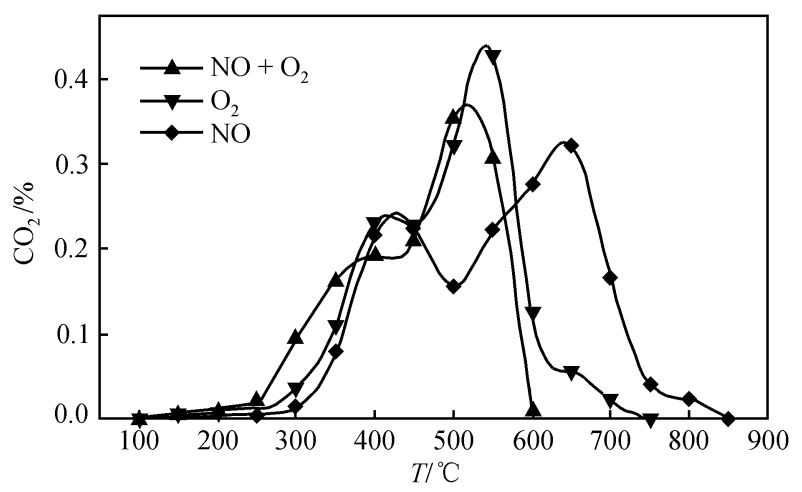

图 $6 \mathrm{Cu}_{0.95} \mathrm{~K}_{0.05} \mathrm{Fe}_{2} \mathrm{O}_{4} / \mathrm{DPF}$ 分别在 $\mathrm{NO}, \mathrm{O}_{2}$ 和 $\mathrm{NO}+\mathrm{O}_{2}$ 情况下进行程序升温反应的 $\mathrm{CO}_{2}$ 浓度比较

在同时去除柴油机 $\mathrm{NO}_{x}$ 和 $\mathrm{PM}$ 的研究中, 一般来 说, 人们期望 $\mathrm{NO} \rightarrow \mathrm{N}_{2}$ 的转化率尽可能高和微粒的燃 烧温度尽可能低. 本研究中碳粒 (soot) 的燃烧温度 $T_{\mathrm{ig}}\left(380^{\circ} \mathrm{C}\right)$ 比原来用碳粒-催化剂混合物进行程序温 
化剂和碳粒的相互接触程度不同而引起的, 对于催 化剂-固体颗粒的反应, 两者相互接触的程度是影响 反应的重要因素 ${ }^{[14]}$. 有关这方面的研究正在进行.

以上研究结果揭示了用 $\mathrm{DPF} /$ 催化方法同时去除 柴油机 $\mathrm{NO}_{x}$ 和 $\mathrm{PM}$ 的有效性. 该技术路线结合了微粒 的捕集、微粒的催化氧化燃烧、 $\mathrm{NO}_{x}$ 被 $\mathrm{SOF}$ 和碳粒 的还原等 3 个过程于一体, 是一种非常有发展前途的 柴油机排放后处理新技术.

致谢本工作为教育部高等学校博士学科点专项科研基 金资助项目(项目编号: 20010248003).

\section{参考文献}

1 Mishima $\mathrm{H}$, Hashimoto $\mathrm{K}$, Ono $\mathrm{T}$, et al. Selective catalytic reduction of $\mathrm{NO}$ with $\mathrm{NH}_{3}$ over natural zeolites and its application to stationary diesel engine exhaust. Appl Catal B, Environmental, 1998, 19: 119 126

2 Koebel M, Elsener M, Kleemann M. Urea-SCR: A promising technique to reduce $\mathrm{NO}_{x}$ emissions from automotive diesel engines. Catal Today, 2000, 59: 335 345

3 Badini C, Saracco G, Serra V, et al. Suitability of some promising soot combustion catalyst for application in diesel exhaust treatment. Appl Catal B, Environmental, 1998, 18: 137 150

4 Miro E E, Ravelli F, Ulla M A, et al. Catalytic combustion of diesel soot on Co, K supported catalysts. Catal Today, 1999, 53: 631 638

5 Neeft J P A, Schipper W, Mul G, et al. Feasibility study towards a $\mathrm{Cu} / \mathrm{K} / \mathrm{Mo} /(\mathrm{Cl})$ soot oxidation catalyst for application in diesel exhaust gases. Appl Catal B, Environmental, 1997, 11: 365 382
6 Yoshida K, Makino S, Sumiya S, et al. Simultaneous Reduction of $\mathrm{NO}_{\mathrm{x}}$ and Particulate Emissions from Diesel Engine Exhaust. SAE Paper 892046, 1989

7 Teraoka Y, Nakano K, Kagawa S, et al. Simultaneous removal of nitrogen oxides and diesel soot particulates catalyzed by perovskite-type oxides. Appl Catal B, 1995, 5: L181 185

8 Shangguan W F, Teraoka Y, Kagawa S. Simultaneous catalytic removal of $\mathrm{NO}_{x}$ and diesel soot particulates over ternary $\mathrm{AB}_{2} \mathrm{O}_{4}$ spinel-type oxides. Appl Catal B, 1996, 8: 217 227

9 Shangguan W F, Teraoka Y, Kagawa S. Kinetics of Soot- $\mathrm{O}_{2}$, Soot-NO and Soot- $\mathrm{O}_{2}$-NO reaction over spinel-type $\mathrm{CuFe}_{2} \mathrm{O}_{4}$ catalyst. Appl Catal B, 1997, 12: 237 247

10 Shangguan W F, Teraoka Y, Kagawa S. Promotion effect of potassium on the catalytic property of $\mathrm{CuFe}_{2} \mathrm{O}_{4}$ for the simultaneous removal of $\mathrm{NO}_{x}$ and diesel soot particulate. Appl Catal B, 1998, 16: 149 154

11 Teraoka Y, Shangguan W, Jansson K, et al. Identification of active crystalline phase in $\mathrm{La}-\mathrm{K}-\mathrm{Cu}-\mathrm{V}$ mixed for the simultaneous removal of nitrogen oxides and diesel soot. Bull Chem Soc Jpn, 1999, 72: 133 137

12 Teraoka Y, Shangguan W F, Kagawa S. Reaction mechanism of simultaneous catalytic removal of $\mathrm{No}_{x}$ and diesel soot particulates res. Chem Intermed, 2000, 26: 201 206

13 Teraoka Y, Nakano K, Shangguan W F, et al. Simultaneous catalytic removal of nitrogen oxide and diesel soot particulate over perovskite-related oxides. Catal Today, 1996, 27: 107 113

14 Neeft J P A, Pruissen O P, Makkee M, et al. Catalysts for the oxidation of soot from diesel exhaust gases, II Contact between soot and catalyst under practical conditions. Appl Catal B, 1997, 12: $21 \sim 31$

(2002-04-24 收稿, 2002-08-17 收修改稿) 